\title{
Design and CFD Analysis of Shock Wave over Supersonic CD Nozzle
}

\author{
Kousik Kumaar. R \\ Assistant Professor, Aeronautical Engg., \\ Nehru Institute of Technology, Coimbatore, India \\ Kesavan. M \\ Assistant Professor, Aeronautical Engg., \\ Nehru Institute of Technology, Coimbatore, India
}

\begin{abstract}
In present work, design of CD nozzle is done and the effect of shock wave is evaluated through CFD analysis. In supersonic flow Shock wave is desirable, but the effect and the operating parameters that move the shock wave into nozzle are crucial. It is analyzed and presented in this research work. NPR value is taken as parameter to analysis the effect.
\end{abstract}

Keywords - Nozzle, CD nozzle, Shockwave, Nozzle Pressure Ratio (NPR)

\section{INTRODUCTION}

Nozzles used for feeding hot blast into a blast furnace or forge are called tubers. Jet nozzles are also use in large rooms where the distribution of air via ceiling diffusers is not possible or not practical. Diffusers that use jet nozzles are called jet diffuser where it will be arranged in the side wall areas in order to distribute air. When the temperature difference between the supply air and the room air changes, the supply air stream is deflected upwards to supply warm air or deflected downwards to supply cold air.

Divergent nozzles slow fluids if the flow is subsonic, but they accelerate sonic or supersonic fluids. Convergent-divergent nozzles can therefore accelerate fluids that have choked in the convergent section to supersonic speeds. This CD process is more efficient than allowing a convergent nozzle to expand supersonically externally. The shape of the divergent section also ensures that the direction of the escaping gases is directly backwards, as any sideways component would not contribute to thrust.

A jet exhaust produces a net thrust from the energy obtained from combusting fuel which is added to the inducted air. This hot air passes through a high speed nozzle, a propelling nozzle, which enormously increases its kinetic energy. Increasing exhaust velocity increases thrust for a given mass flow, but matching the exhaust velocity to the air speed provides the best energy efficiency. However, momentum considerations prevent jet aircraft from maintaining velocity while exceeding their exhaust jet speed. The engines of supersonic jet aircraft, such as those of fighters and SST aircraft (e.g. Concorde) almost always achieve the high exhaust speeds therefore necessary for supersonic flight by using a CD nozzle despite weight and cost penalties; conversely, subsonic jet engines employ relatively low, subsonic, exhaust velocities and therefore employ simple convergent nozzle, or even bypass nozzles at even lower speeds.

\section{DE-LAVAL NOZZLE}

A de Laval nozzle will only choke at the throat if the pressure and mass flow through the nozzle is sufficient to reach sonic speeds, otherwise no supersonic flow is achieved and it will act as a venture tube; this requires the entry pressure to the nozzle to be significantly above ambient at all times (equivalently, the stagnation pressure of the jet must be above ambient). In addition, the pressure of the gas at the exit of the expansion portion of the exhaust of a nozzle must not be too low. Because pressure cannot travel upstream through the supersonic flow, the exit pressure can be significantly below ambient pressure it exhausts into, but if it is too far below ambient, then the flow will cease to be supersonic, or the flow will separate within the expansion portion of the nozzle, forming an unstable jet that may 'flop' around within the nozzle, possibly damaging it. In practice ambient pressure must be no higher than roughly 2-3 times the pressure in the supersonic gas at the exit for supersonic flow to leave the nozzle. 


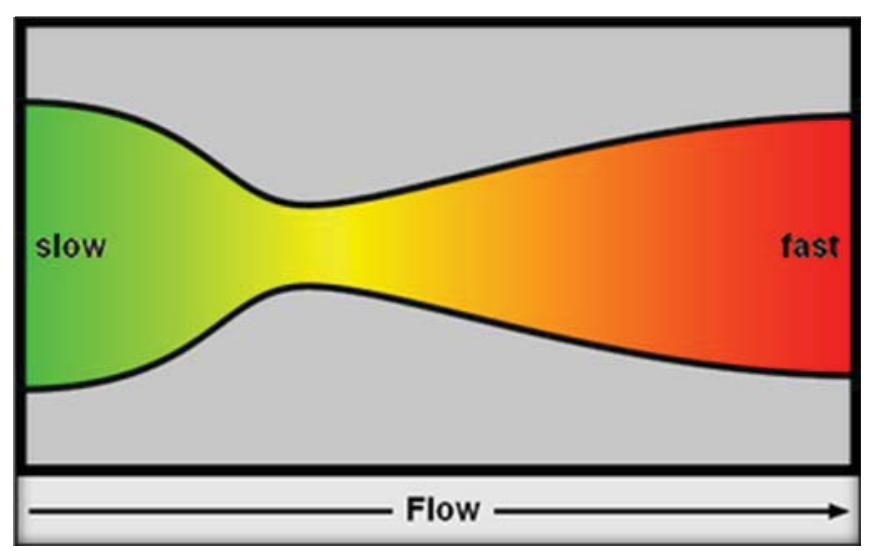

Fig. 1.1 De-laval Nozzle

\section{LITERATURE REVIEW}

M. Al-Ajlouni presented two computational stages have been used and both with the aid of the computer. Firstly, a well-known calculation and graphical packages have been used to create the unit model matrix for each Mach number. Then, in the second stage, programming with visual basic has been used to create the profile of the nozzle automatically by multiplying the unit model matrix by the proper scale factor that is calculated according to the working requirements. User friendly software has been written for this purpose. The functionality of the method is validated by solving problems with known solutions and this approach presented logical results. This work is limited for Mach number up to 2.5 only, constant specific heat and two dimensional flows [1].

Hasan Ali et. al presents complete design philosophy is developed and implemented for the ideal conditions. To address the actual conditions, the consideration should be made on account of the phenomena due to the viscous effect, pressure difference with respect to the back pressure, heat conduction and so on. The design presented here can be utilized to compare with the other nozzle designs regarding to the specific design conditions. The simulation program of the practical supersonic nozzle can be developed depending on this design with the considerations of the losses take place in real time [2].

Blom Martina has given numerical investigation of $3 \mathrm{~d}$ compressible duct flow a generic supersonic bump in a duct has been studied. The shock wave over the bump is accelerated, inducing a shock wave in the downstream, interacting with the wall boundary layer and triggering boundary layer separation [3].

Gustavo Bono et. al presented an adaptive mesh strategy based on nodal re-allocation is presented in this work. This technique is applied to problems involving compressible flows with strong shocks waves, improving the accuracy and efficiency of the numerical solution. The initial mesh is continuously adapted during the solution process keeping, as much as possible, mesh smoothness and local orthogonality using an unconstrained nonlinear optimization method. The adaptive procedure, which is coupled to an edge-based error estimate aiming to equidistribute the error over the cell edges is the main contribution of this work. The flow is simulated using the Finite Element Method (FEM) with an explicit one-step Taylor- Galerkin scheme, in which an Arbitrary LagrangeanEulerian (ALE) description is employed to take into account mesh movement. Finally, to demonstrate the capabilities of the adaptive process, several examples of compressible in-viscid flows are presented [5].

S. Carcano investigated the decompression structure inside a gas-particle under expanded jet and we have analysed the non-equilibrium effects between the gas phase and the solid particulate phase. The objective has been to identify different regimes and to quantify non-equilibrium effects as a function of different characteristic time scales of the supersonic jet and the solid particles. A ratio $r$ between the particle relaxation time and the characteristic jet time scale has been introduced. This ratio allows identifying a pseudo gas regime, in which non-equilibrium effects between the gas and the solid phase are negligible. Moreover, the value of the parameter is related to the change in the decompression structure of the under-expanded jet, in particular to the location and the intensity of the standing normal shock. The analysis has been developed for the case of mono-disperse mixtures and then extended to the case of bi-disperse and poly-disperse mixtures of gas and solid particles [6]. 
Giglmaier M. Presents that a novel method of nano-particle production by shock-induced ignition of a precursor and aerodynamic quenching, developed within a DFG founded project, is the focus of the present study. The reduction of the shock/boundary layer interaction in order to obtain homogeneous post-shock conditions is necessary for a narrow size distribution of the produced particles. Due to a required mixing length and a smooth supersonic nozzle, the boundary layer in our nano-particle reactor thickens up and reaches about $20 \%$ of the channel height. Additionally, due to the multiple boundary layer interactions the single shock disintegrates into a so called pseudo-shock. Thereby, oblique shocks form an aerodynamic nozzle with regions of compressions and expansions which results in inhomogeneous downstream conditions. These effects can be reduced by active and passive control techniques which are compared within this investigation [13].

Esam I. Jassim et. al presents Separation of undesirable particles from natural gas flow using supersonic nozzle is a promised technology that turns oil and gas firm's attention to enhance the already existing systems. Utilizing supersonic nozzle for this purpose has showed a positive impact on the separation technology due to simplicity in designing, cost effective in manufacturing, and feasibility in maintenance The research in this work focus mainly on employing CFD commercial software to study the influence of nozzle shape on the shockwave location since such location impacts the turbulence of the flow that eventually forces small particles to move toward the nozzle wall. Hence, improves the collection efficiency. The numerical results show that nozzle shape is slightly changed with the shape of the nozzle. Elliptical Nozzle predicts shockwave a bit later than other shapes for specific NPR. However at high NPR hexagon nozzle is the one among the rest whose shock location becomes the farthest from the nozzle throat [14].

Ali kargar's work is devoted to numerical analysis of supersonic- subsonic flow in the convergent- divergent nozzle along with the shock and comparison with analytical results. Shock occurrence point is considered, and its results are compared with analytical amounts. Governing equations written in conservation form and artificial nonviscosity term is used to prevent high vibrations in shock area in momentum equation. Because of changing cross section along the flow, all fluid characteristics would be considered a function of $\mathrm{x}$. so by choosing an appropriate control volume, the main equations include continuity, momentum and energy were discrete .To solve the main equations, McCormack explicit method are used [15].

Kunal Pansari et. al presented analysis of the performance and flow characteristics of convergent divergent (c-d) nozzle. We can conclude that the shock strength goes on increasing with Decreasing operating pressure ratio and also the shock location move towards exit. Exit Mach number (Me) and Mach number ahead of the shock (M1) goes on increasing by decreasing the operating pressure ratio. A numerical study has been carried out to analyse the performance and flow characteristics of the convergent-divergent nozzle under various operating pressure ratio and with different nozzle profiles, also to determine the location and strength of the normal shock in the divergent portion of the nozzle [21].

O. Thomer et. al said that the interaction of slender vortices with normal and oblique shocks is investigated for a Mach number of 1.6. The conservation equations for three-dimensional, time dependent, in viscid and viscous flows of ideal gases are solved numerically. At the inflow boundary a Burgers vortex is prescribed with variable circulation and axial velocity distribution. The calculations performed on meshes with approximately 2 million grid points show that the breakdown is primarily controlled by pressure and therefore occurs more easily when a vortex interacts with a normal shock. Oblique shocks are deformed into an ' $S$ '-shaped part near the vortex core where the shock angle is increased. Downstream from the shock the velocity is locally subsonic. The results indicate that initiation of breakdown is much more sensitive to variations in the axial velocity than in the circulation [25].

Q. Xiao presents separation of supersonic flow in a planar convergent-divergent nozzle with moderate expansion ratio is investigated by solving the Reynolds-averaged Navier-Stokes equations with a two-equation turbulence model. The focus of the study is on the structure of the fluid and wave phenomena associated with the flow separation. Computations are conducted for an exit-to-throat area ratio of 1.5 and for a range of nozzle pressure ratios. The results are compared with available experimental data in a nozzle of the same geometry. The flow separates by the action of a lambda shock, followed by a succession of expansion and compression waves. For 1:5 $<$ NPR $<2: 4$, the computation reveals the possibility of asymmetric flow structure. The computationally obtained asymmetric flow structures are consistent with previous experimental flow visualizations studies. In addition, other flow features such as shock location and wall pressure distributions are also in good agreement with the experimental data. The present study provides new information that confirms earlier conjectures on the flow-wave structure relevant to the instability of the separated flow in convergent-divergent nozzles of moderate expansion ratio [27]. 


\section{EXPERIMENTAL WORK}

\subsection{DESIGN CALCULATIONS}

\section{1. a. Input Parameters}

Table 3.1. Input Parameters

\begin{tabular}{|c|c|}
\hline $\mathrm{P}_{1}$ & $1737653 \mathrm{~Pa}$ \\
\hline $\mathrm{P}_{\text {exit }}$ & $101325 \mathrm{~Pa}$ \\
\hline $\mathrm{T} 1$ & $473 \mathrm{~K}$ \\
\hline $\mathrm{R}$ & $287 \mathrm{~J} / \mathrm{Kg} \cdot \mathrm{K}(\mathrm{Gas}$ Constant $)$ \\
\hline Mass flow rate & $32.24 \mathrm{Kg} / \mathrm{s}$ \\
\hline $\mathrm{d}_{1}$ & $140 \mathrm{~mm}$ \\
\hline Fluid & Ideal Gas \\
\hline
\end{tabular}

\section{1. b. Nozzle Design}

Ldiv nozzle (length of divergent nozzle $)=\left(\right.$ dexit $\left.-\mathrm{d}^{*}\right) / 2 * \tan \mathrm{d}$

$$
=105.39 \mathrm{~mm}
$$

$\alpha \mathrm{d}-$ Divergent angle $=18.5^{\circ}$

Lcov nozzle $($ Length of convergent nozzle $)=\left(\mathrm{d} 1-\mathrm{d}^{*}\right) / 2 * \tan \alpha \mathrm{c}$

$$
=58.04 \mathrm{~mm}
$$

$\alpha \mathrm{c}-$ Convergent angle $=13.2^{\circ}$

\section{1. c. CAD Model}

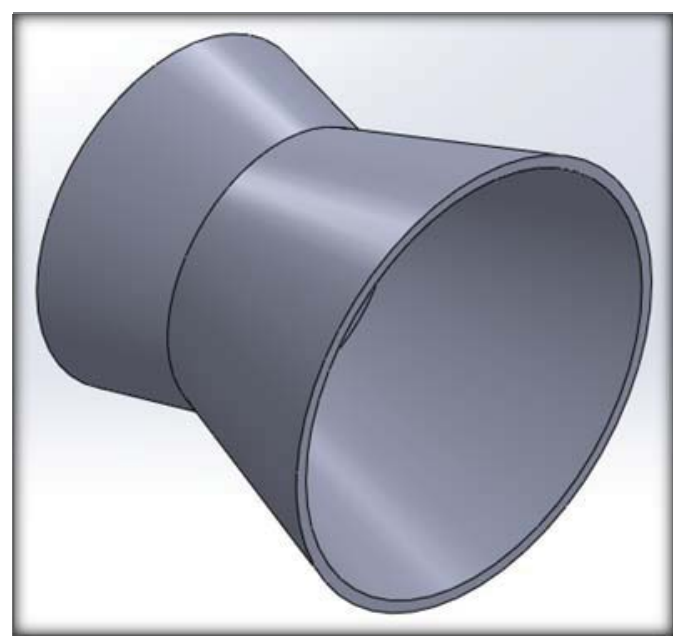

Fig. 3.1 CD-Nozzle 


\subsection{Meshing}

Fluid Cells - 8623

Solid Cells - 4008

Partial Cells - 9262

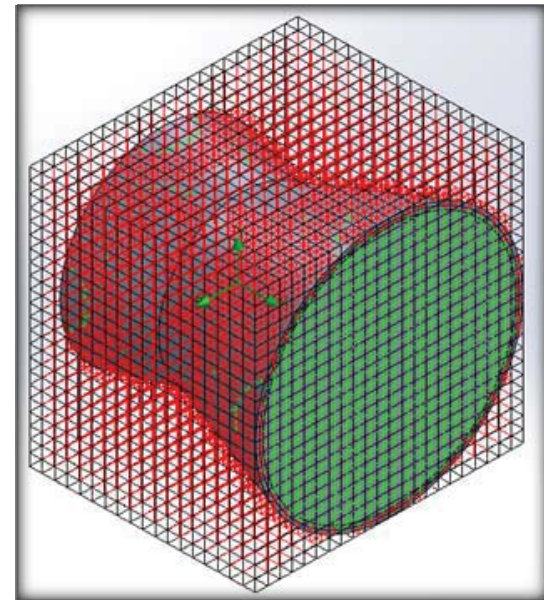

Fig. 3.2 Meshing

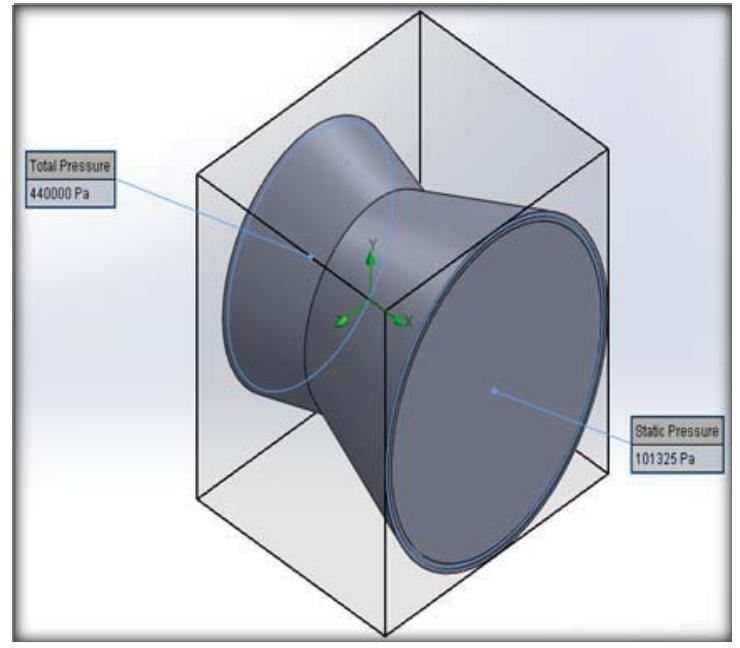

Fig. 3.3 Computational Domain and Boundary Condition

\subsection{SIMULATION RESULTS OF PRESSURE AND TEMPERATURE}
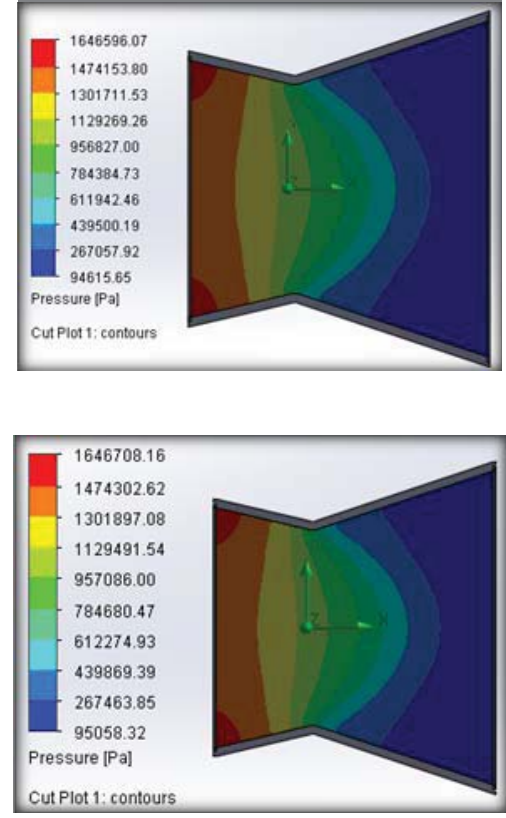

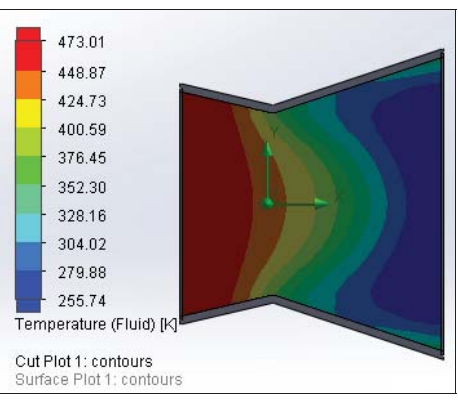

Fig. 3.4 NPR of 10.17

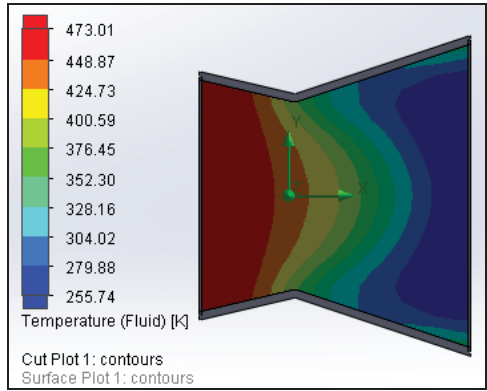

Fig. 3.5 NPR of 7.64 

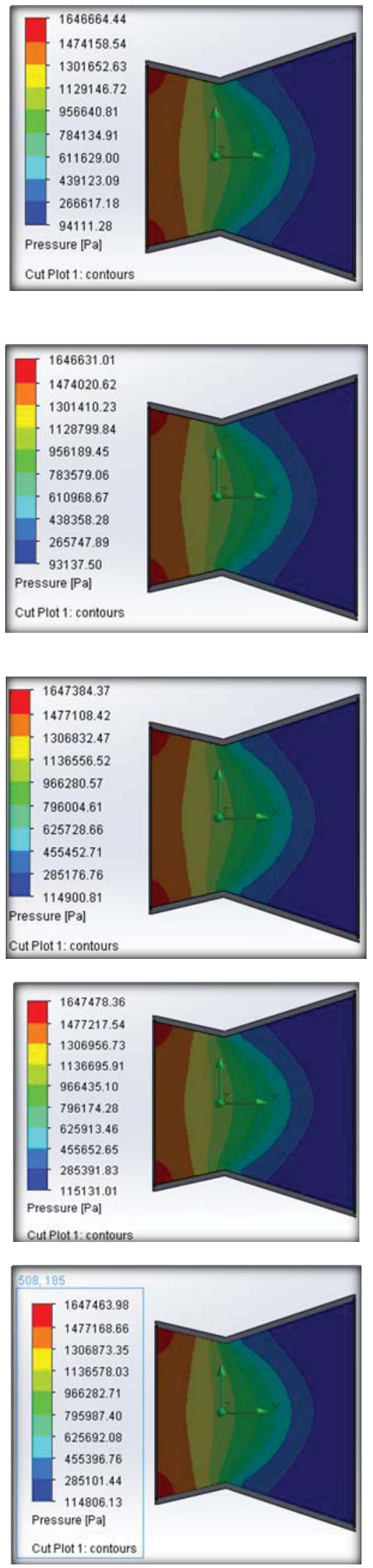

Fig. 3.6 NPR of 6.13
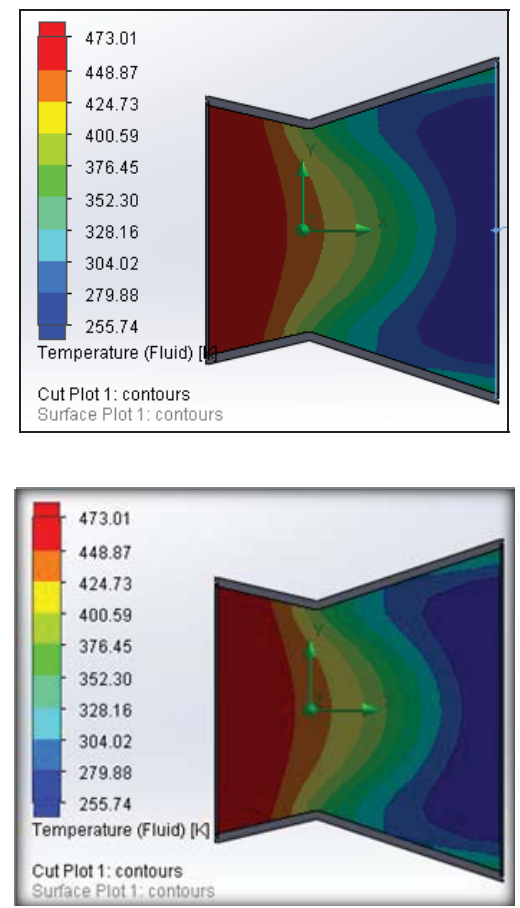

Fig. 3.7 NPR of 5.12

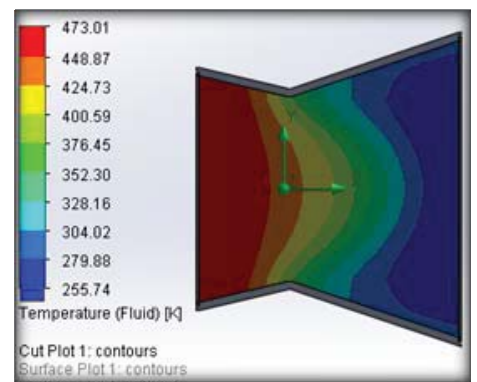

Fig. 3.8 NPR of 3.66

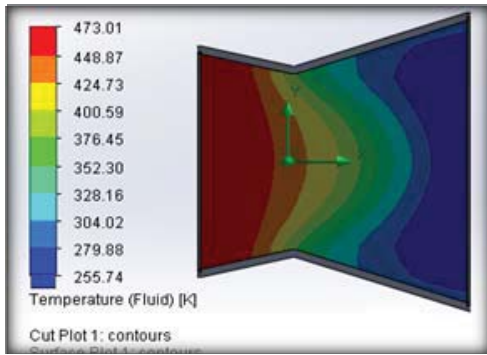

Fig. 3.9 NPR of 2.42

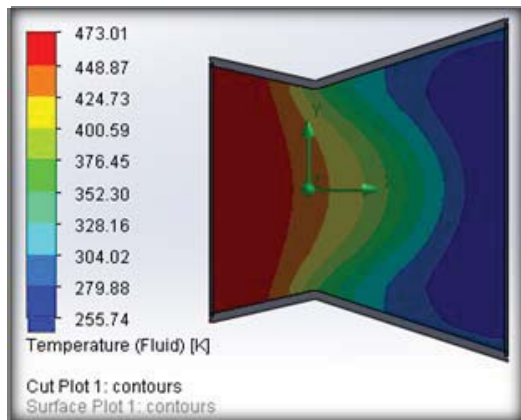

Fig. 3.10 NPR of 1.8 

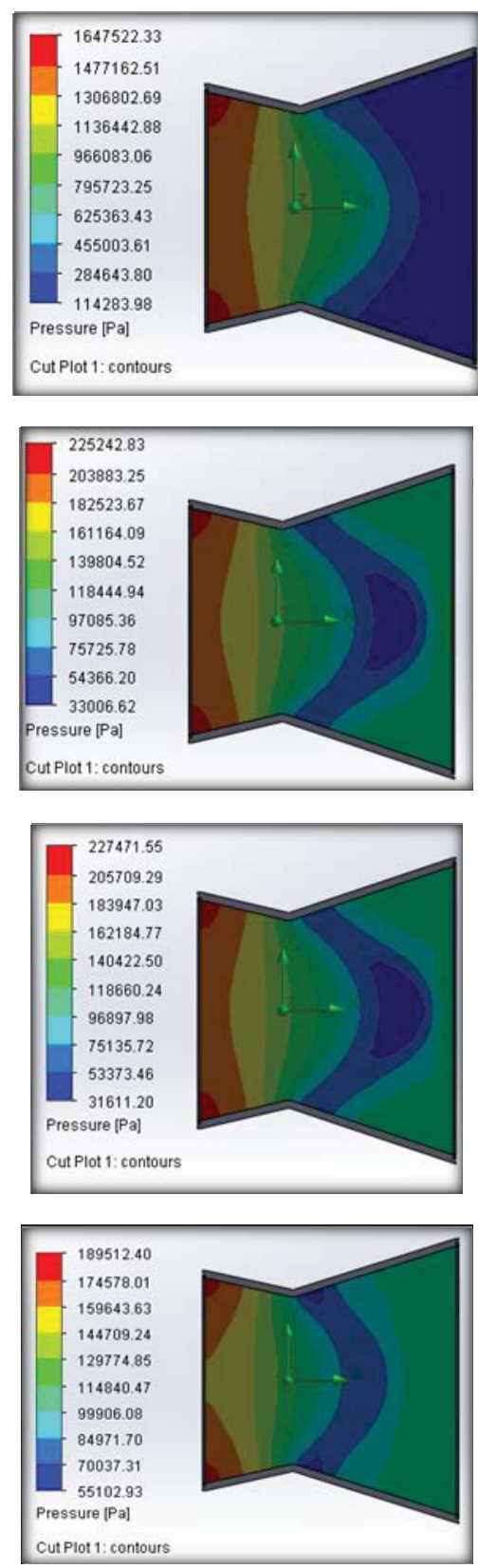

Fig. 3.13 NPR of 1.003

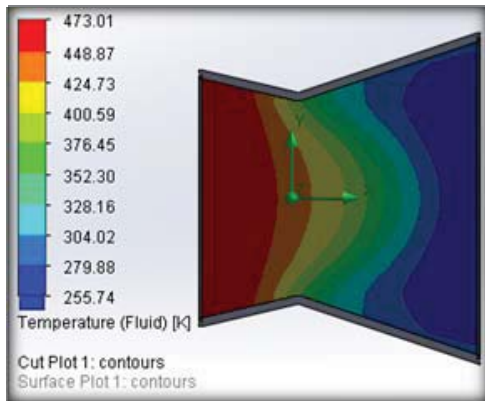

Fig. 3.11 NPR of 1.1

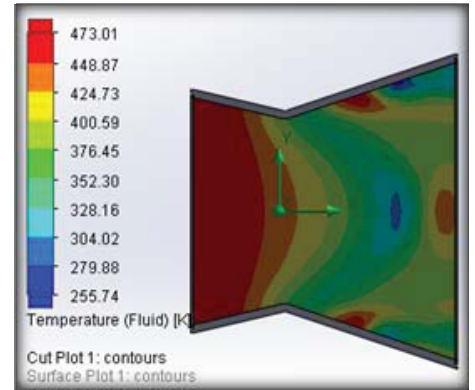

Fig. 3.12 NPR of 1.01
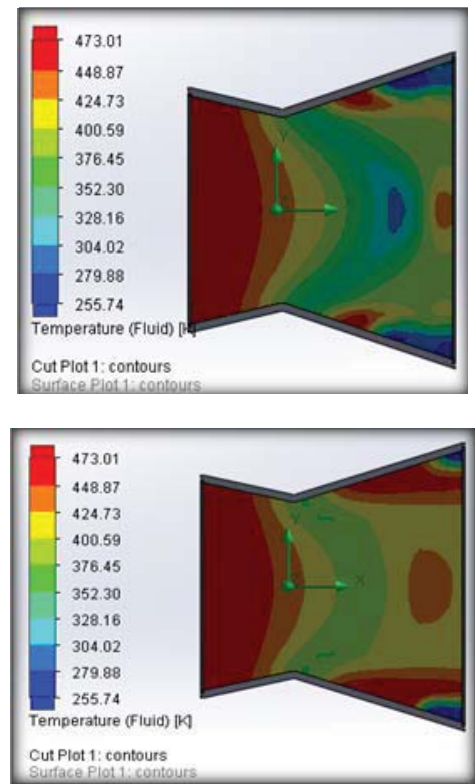

Fig. 3.14 NPR of 1.002 (2 bar -Inlet Pressure)

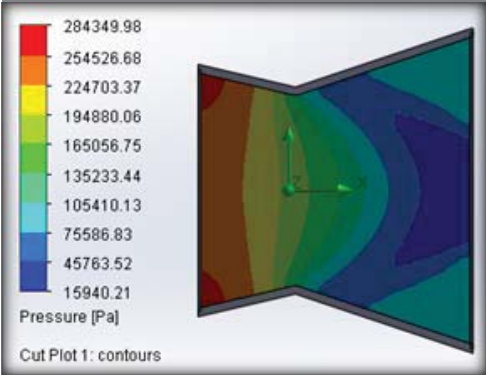

Fig. 3.15 NPR of 0.858 (3 bar-Inlet Pressure) 

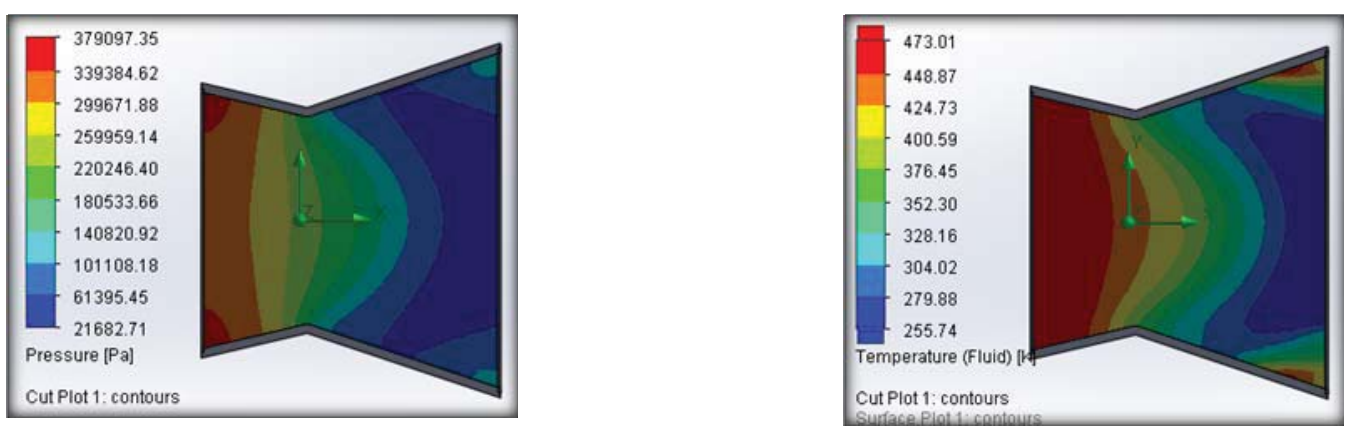

Fig. 3.16 NPR of 0.689 (4 bar-Inlet Pressure)

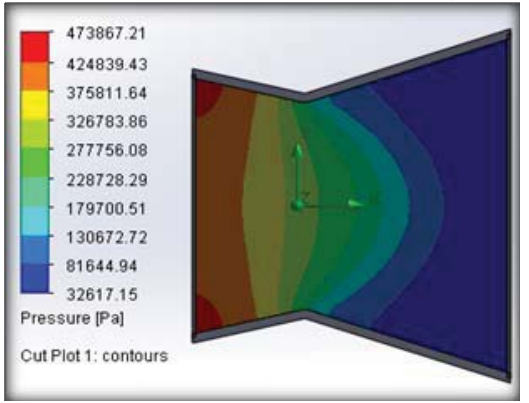

Fig. 3.17 NPR of 0.407 (5 bar-Inlet Pressure)

\section{SUMMARY AND CONCLUSIONS}

Nozzle is designed for 17 bar inlet pressure to 1 bar ambient pressure approximately. In simulation results, outlet pressure of nozzle for above designed condition is 1.42 bar. 1 bar is ambient condition taken in design to match nozzle outlet pressure to 1 bar (NPR = 1). It is perfectly expanded nozzle. There will be always a variation from theoretical design to actual working condition. It is showed in simulation results. Here, we have designed a new CD Nozzle and simulated for different boundary conditions. Inlet and ambient boundary conditions are varied from $17-2$ bar and $1-0.15$ bar respectively to achieve different NPR values. In theory, NPR value is important factor, based on which shockwave effects are studied.

In our case, shockwave comes when NPR value is reduced from 1.02 to 0.5 . Shock wave is tried to come inside of nozzle from inlet pressure of 4.4 bar to outlet pressure of 1 bar. It continuously comes in and chocks the nozzle at 2 bar to 1 bar. In between that, different NPR is achieved from 1.02 to 0.5 . There is a notable thing, at 9 bar inlet and 1 bar ambient pressure, $0.76 \mathrm{NPR}$ is achieved. At that time, no shock wave comes inside of nozzle. Therefore, ratio of inlet pressure to ambient pressure plays an important role in shockwave effects.

In this work, $\mathrm{CD}$ nozzle is designed for a design point and simulated for different NPR values at different inlet and ambient pressures. It is studied for shockwave effects and their influences over downstream flow temperature. Temperature is increased in downstream of shockwave. Shockwave comes inside of nozzle from NPR of 1.02 to 0.568 . There is an important finding in this work. Shockwave travel from outside to inside of nozzle is not only influenced by NPR but also by inlet pressure of nozzle. If ratio between nozzle inlet pressure and outlet pressure is more, it prevents shockwave to come in.

\section{REFERENCES}

[1] Al-Ajlouni M. (2010) 'An Automaic Method for Creating the Profile of Supersonic Convergent-Divergent Nozzle', Jordan Journal of Mechanical and Industrial Engineering, Volume 4, Number 3, Pages $404-411$.

[2] Ali H.M. et. al (2012) 'Numerical Solution For The Design Of Minimum Length Supersonic Nozzle', ARPN Journal of Engineering and Applied Sciences, vol. 7, no. 5, pg: 602-615.

[3] BLOM M. (2010) 'Numerical Investigation of 3D Compressible Duct Flow', Chalmers University of Technology, Goteborg, Sweden.

[4] Burak O. et. al (2009) 'Experimental and numerical investigation of a supersonic C-D nozzle', AIAA, pg: 1-25.

[5] Bono G. et. al (2008) 'An Adaptive Mesh Strategy for High Compressible Flows Based on Nodal Re-Allocation', J. of the Braz. Soc. of Mech. Sci. \& Eng, No. 3 / 189 
[6] Carcano S. (2013) 'Numerical investigation of interaction between shock waves and dusty flows', Second ECCOMAS Young Investigators Conference 2-6 September 2013, Bordeaux, France.

[7] Chuech S.G. et. al (1989) 'Structure of Turbulent Sonic Under-expanded Free Jets', AIAA JOURNAL, VOL. 27, NO. 5, Pg: 549-559.

[8] Chen J. et. al (2007) 'Investigation On The Pressure Matching Performance Of The Constant Area Supersonic-Supersonic Ejector', IJERT, vol. 5, no. 5, pg: 7-16.

[9] Das S. et. al (2010) 'Behaviour Of Flow Unsteadiness in A Supersonic Air-Intake With Throttles', Proceedings of the 37th International \& 4th National Conference on Fluid Mechanics and Fluid Power.

[10] Emmert T. et. al (2006) 'Numerical Study of Aero Acoustic Oscillations in Transonic Flow Downstream a Sudden Duct Enlargement', 27th AIAA Aero Acoustics Conference.

[11] Foutter R. (1995) 'A numerical investigation of weak shock wave effects in reacting nozzle flows'.

[12] Gerritsma M. et. al (2008) 'Least-Squares Spectral Element Methods For Compressible Flows', 6th International Conference on Heat Transfer, Fluid Mechanics and Thermodynamics.

[13] Giglmaier M. et. al (2008) ' Gasdynamic Optimization of a Transonic Nano-particle Reactor - Numerical Investigation of Active/Passive Shock Control', Joint Advanced Student School, Saint Petersburg, Modelling and Simulation in Multidisciplinary Engineering.

[14] Jassim E.I. et. al (2013) 'Numerical Investigation of Nozzle Shape Effect on Shock Wave in Natural Gas Processing', World Academy of Science, Engineering and Technology, Vol - 7

[15] Kargar A. et. al (2009) 'Numerical analysis of supersonic- subsonic flow in the convergent- divergent nozzle along with the shock and comparison with analytical results', vol-2, no.4.

[16] KUMAR K.V. et. al (2011) 'Exploration of Supersonic Flow Through Conical Nozzle With Diverse Angles Of Divergence', International Journal of Latest Trends in Engineering, Science \& Technology Volume 1, Issue 1, pg: 2-9.

[17] Kudryavtsev A.N. et. al (2002) 'Numerical Investigation of Shockwave Interactions in Supersonic Imperfectly Expanded Jets', vol 3, no. 3, pg: 4-11.

[18] Kremeyer K. et. al (2006) 'Computational Study of Shock Mitigation and Drag Reduction by Pulsed Energy Lines', AIAA Journal, Vol. 44, No. 8, pg: 1720-1731.

[19] Noh M.H.M. et. al (2011) 'Numerical Investigation of Chocked Converging-Diverging Nozzles For Thruster Application', IIUM Engineering Journal, Special Issue, Mechanical Engineering.

[20] Pansari K. et. al (2013) 'Analysis Of The Performance And Flow Characteristics Of Convergent Divergent (C-D) Nozzle', International Journal of Advances in Engineering \& Technology, Vol. 6, Issue 3, pp. 1313-1318.

[21] Pansari K. et. al (2013) ' Numerical Investigation of the Perfomance of Convergent Divergent Nozzle', International Journal of Modern Engineering Research, Vol. 3, Issue. 5, pp-2662-2666.

[22] Pandey K.M. et. al (2010) ' Recent Advances in Experimental and Numerical Analysis of Combustor Flow Fields in Supersonic Flow Regime', International Journal of Chemical Engineering and Applications, Vol. 1, No. 2, pg: 132-137.

[23] Rao G.R. et. al (2013) 'Flow Analysis in a Convergent-Divergent Nozzle Using CFD’, IJRME, vol 1, Issue 2, pg: 136-144.

[24] Rahul R.V. et. al (2002) 'Unsteady computation of overexpanded flow in convergent divergent nozzle', ICCCSA.

[25] Thomer O. et. al (2000) 'Computational Study Of Normal And Oblique Shock-Vortex Interactions', European Congress on Computational Methods in Applied Sciences and Engineering, pg: 1-20.

[26] Throckmorton R. et. al (2007) 'Experimental and Computational Investigation of a Dynamic Starting Method for Supersonic/Hypersonic Inlets', American Institute of Aeronautics and Astronautics.

[27] Xiao Q. et. al (2007) 'Numerical Investigation of Supersonic Nozzle Flow Separation', AIAA Journal, Vol. 45, No. 3, pg: $532-541$.

[28] Yi K.J. et. al (2011) 'Prediction of the Rear Fuselage Temperature with Radiation Shield', World Academy of Science, Engineering and Technology Vol:5, pg: 1044-1047. 